\title{
Considerations for Takayasu Arteritis Undergoing General Anesthesia in a Resource-Limited Setting: Case Report and Literature Review
}

\author{
Corinna Ongaigui, $M D^{*}$ and Rosalina B Anasarias, $M D$
}

Department of Anesthesiology, East Avenue Medical Center, Quezon City, Philippines

\begin{abstract}
Working in a resource-limited setting in terms of equipment and medication choices is a recurring challenge for anesthesiologists from low-to middle-income countries. Experts in global health highlight the importance of providing safe anesthesia care practices such as optimizing nontechnical skills like cognitive decision-making, planning and versatile resource management. In this report, we present a case of a 23-year-old male with an incidental preoperative diagnosis of Takayasu arteritis who had undergone successful surgery under general anesthesia in a resource-limited setting. In addition, we discuss what the perioperative considerations are for this patient population.
\end{abstract}

Keywords

Takayasu arteritis, General Anesthesia, Anesthetic management

\section{Introduction}

Takayasu arteritis, also called "aortic arch syndrome", "young female arteritis", and "pulseless disease", is a rare chronic progressive large-vessel vasculitis of unknown etiology that affects the aorta and its major branches [1]. The clinical presentation of Takayasu arteritis patients vary greatly, which often makes definitive diagnosis difficult and delayed. The disease predominantly affects Asian women during the second or third decade of life and is associated with considerable morbidity and premature mortality attributed to a delay in diagnosis $[2,3]$. The anesthetic management of patients with Takayasu arteritis is well described with most of the literature involving patients who had undergone surgery under neuraxial anesthesia [4-10]. Furthermore, reports have emphasized the importance of utilizing intraoperative monitoring devices such as invasive arterial blood pressure and cerebral perfusion monitoring devices [10-12], which are unavailable in our institution when this case was performed. We therefore present our experience of a patient with Takayasu arteritis who had undergone general anesthesia and further discuss the anesthetic considerations associated with Takayasu arteritis in a resource-limited setting.

\section{Case Report}

A 23-year-old man (height, $163 \mathrm{~cm}$; weight, $37 \mathrm{~kg}$; BMI, 13.9) was scheduled for endoscopy-guided turbinoplasty for septal deviation with turbinoseptal contact of the right middle turbinate and posterior area of the nasal septum. His history of present illness started nine months prior to surgery when the patient consulted an otorhinolaryngologist due to frequent episodes of nasal congestion accompanied by pain between the eyes, headache and dizziness that were not relieved by oral medication. After undergoing panendoscopy, the doctor attributed his symptoms to a deviated nasal septum and advised turbinoplasty. His past medical history only consisted of an uncomplicated hemorrhoidectomy done in 2011. He was an otherwise healthy man with unremarkable personal and social history and no family history of anesthetic problems or other inheritable conditions.

On physical examination by the attending physician, it was noted that his pulse was feeble on the left arm precluding blood pressure measurement using a conventional sphygmomanometer and stethoscope. On the other hand, pulses were audible on his right arm and his blood pressure (BP) was noted to be $100 / 60 \mathrm{mmHg}$. Laboratory findings were unremarkable. Twelve-lead ECG revealed left ventricular hypertrophy and 2-D echocardiography showed mild regurgitation and mild

*Corresponding author: Corinna Ongaigui, MD, Department of Anesthesiology, East Avenue Medical Center, Quezon City, NCR, Philippines

Accepted: January 20, 2020

Published online: January 22, 2020

Citation: Ongaigui C, Anasarias RB (2020) Considerations for Takayasu Arteritis Undergoing General Anesthesia in a Resource-Limited Setting: Case Report and Literature Review. J Clin Anesth Pain Manag 4(1):88-91 
Citation: Ongaigui C, Anasarias RB (2020) Considerations for Takayasu Arteritis Undergoing General Anesthesia in a Resource-Limited Setting: Case Report and Literature Review. J Clin Anesth Pain Manag 4(1):88-91

mitral valve prolapse with an ejection fraction of $69 \%$. The patient was referred to a cardiologist who observed similar physical examination findings in addition to a decreased left carotid pulse.

A computed tomographic angiography was done which revealed an occlusion of the left common carotid artery and left subclavian artery. There is a short segment stenosis on the proximal celiac artery, as well as double renal artery supply of the right kidney, an ectatic left renal artery, and a left-sided inferior vena cava in the abdominal region. The aorta was found to be normal with no evidence of stenosis, aneurysm, or dissection. The brachiocephalic artery, right common carotid artery and right subclavian artery were patent. The patient was subsequently diagnosed with Takayasu arteritis and he was started on a combination therapy consisting of prednisolone, methotrexate and folic acid.

Before surgery, the patient was premedicated with oral midazolam. Once in the operating theater, automated noninvasive BP monitors were placed on the patient's right upper and lower limbs. Electrocardiogram (5-lead) and finger pulse oximeter were also placed. Baseline BP of $100 / 60 \mathrm{mmHg}$ on the right upper limb and $120 / 70 \mathrm{mmHg}$ on the right lower limb were recorded before the induction of anesthesia.

After proper preoxygenation, general anesthesia was induced using intravenous midazolam $1 \mathrm{mg}$, fentanyl $50 \mathrm{mcg}$, lidocaine $20 \mathrm{mg}$, and propofol $100 \mathrm{mg}$. This was followed by administration of intravenous atracurium $25 \mathrm{mg}$ and oral intubation was performed using a McCoy blade laryngoscope. Anesthesia was maintained with sevoflurane $1.5 \%$ with oxygen and intermittent boluses of phenylephrine were given as needed to maintain mean arterial pressure within $20 \%$ of the preoperative values. He was also given an intravenous dose of acetaminophen $1 \mathrm{~g}$ and tramadol $50 \mathrm{mg}$ as preventive analgesia. The patient remained hemodynamically stable throughout the 2-hour procedure, sustaining only minimal blood loss. After recovery of neuromuscular blockade and adequate spontaneous breathing, the patient was given intravenous lidocaine $80 \mathrm{mg}$ prior to deep extubation. The entire postoperative course was uneventful and the patient was discharged on the fourth postoperative day.

\section{Discussion}

Takayasu arteritis is a rare chronic intractable disease of unknown etiology characterized by vasculitis involving mainly the aorta and its major branches. It is the arterial inflammation that causes stenosis, occlusions or aneurysmal dilatations that predispose the patient to symptomatic end-organ ischemia [13-15]. The clinical presentation of patients with Takayasu arteritis, therefore, varies greatly depending on the extent of vascular involvement and the degree of disease progression. Considerable morbidity and premature mortality are attributed to a delay in diagnosis; thus, a high index of clinical suspicion is emphasized $[2,14,16]$.

As for epidemiology, Takayasu arteritis is most commonly seen in Japan, South East Asia, and India [17]. In the Philip- pines, the reported combined number of cases of Takayasu arteritis at the University of the Philippines-Philippine General Hospital and Philippine Heart Center is 61 during the years 2006-2015 [18]. It is now recognized worldwide that the disease can affect both sexes and may have an onset of $>$ 40 years of age $[19,20]$. Moreover, a recent study reported a larger proportion of Takayasu arteritis patients who are male and with an elderly onset [14]. However, the disease may also present in childhood [21,22].

Classification of the disease can be radiographic based on angiographic findings or clinical based on symptoms or complications [23,24]. Ishikawa [23] described the four most important complications as secondary hypertension, retinopathy, aneurysm formation, and aortic regurgitation. Hypertension is the most common complication observed in a Filipino multicenter cohort [18].

Cited descriptions on the anesthetic management of patients with Takayasu arteritis were mostly of parturient women who have undergone successful neuraxial anesthesia $[8,9,25-28]$. Irrespective of the choice of anesthetic technique, pre-anesthetic assessment must initially take into account the extent of affected arteries and degree of organ involvement, as intraoperative anesthetic concerns are often related to severe uncontrolled hypertension, stenosis or aneurysms of blood vessels that may affect regional circulation predisposing to end-organ dysfunction, and difficulties in monitoring the blood pressure [11,29]. A history of dizziness or syncope on extension of the head may be a clinical feature suggestive of carotid involvement that compromises cerebral perfusion; hence, is an important consideration when laryngoscopy is contemplated $[7,11]$.

Preoperatively, standard ASA monitoring is adequate for most of these patients $[7,11,26,28,30]$. The use of intraoperative cerebral perfusion monitoring devices has been found to be of clinical value to detect ischemia among patients with cerebral artery stenosis [10,31-33]. This is important as postoperative cerebral infarction can occur in this patient population [34]. Hypertensive parturient women are especially vulnerable and the use of regional cerebral oxygen saturation guided cerebral monitoring during delivery has been described [35]. Nonetheless, in patients with undocumented cerebrovascular defects, there have been several reports of uncomplicated neurological outcomes in which general anesthesia was used without these devices $[7,11,12,21,30,33,36]$.

The key concept of perioperative anesthetic management is to maintain adequate blood flow to vital organs by avoiding significant blood pressure fluctuations especially severe hypertension. Both noninvasive and invasive blood pressure monitoring have been described in literature $[11,12,21,26,27,32-34,36-39]$. Blood pressure is best measured from the extremity least affected by the disease process. Monitoring of upper and lower extremities is advocated only when there is a discrepancy of more than 20 $\mathrm{mmHg}[7,25]$. Meikli and Milne, however, suggested that monitoring of both upper and lower blood pressure should be considered to have some measure of overall perfusion 
Citation: Ongaigui C, Anasarias RB (2020) Considerations for Takayasu Arteritis Undergoing General Anesthesia in a Resource-Limited Setting: Case Report and Literature Review. J Clin Anesth Pain Manag 4(1):88-91

[40]. Other methods of noninvasive blood pressure monitoring that have been reported include the use of Doppler blood flow signals and visual estimation of systolic blood pressure using plethysmographic waveform by pulse oximetry $[29,41]$. Intra-arterial monitoring of blood pressure may be necessary if extreme and rapid blood pressure fluctuations are anticipated from the surgery or if no blood pressure recordings are attainable from any extremity $[12,21,33,36]$. Another study recommended monitoring of beat-to-beat variations in blood pressure as a proactive measure to correct mean arterial pressure intraoperatively [27]. It is further advised that in inserting the catheter, the tip should be placed proximal to the area of arteritis, as there is a difference in blood pressure proximal and distal to the area of arteritis. Central system blood pressure was reported to be 100 to $120 \mathrm{mmHg}$ over and above those obtained in the upper limbs peripherally [42].

Because the patient has an occlusion on the left main arteries of the neck, we used a McCoy blade laryngoscope to intubate the patient to reduce the head extension angle that may predispose the patient to cerebral ischemia. The McCoy laryngoscope, introduced in 1993, is a modification of the Macintosh laryngoscope. It is a levering laryngoscope that has a hinged tip that is operated by a level mechanism on the back of the handle which permits epiglottic elevation [43]. In hospitals where fiberoptic bronchoscopes or video laryngoscopes are not available, the McCoy laryngoscope is a useful alternative in visualizing the airway in patients with limited neck extension [44]. In our case, the attending anesthesiologist happened to own a McCoy laryngoscope. In cases where a McCoy laryngoscope is unavailable, intubation can still be achieved using a Macintosh laryngoscope [33]. Although limited evidence suggests that head extension during intubation in patients with cervical disorders may cause secondary injuries [45], a prompt smooth intubation is advised as it can also help prevent prolonged unwanted hemodynamic stress responses that can be detrimental to high-risk patients with Takayasu arteritis.

Postoperatively, means to avoid hypertension associated with extubation and acute pain should be taken. Moreover, postoperative pain relief is essential to prevent undesirable stress-related complications. The decision to transfer the patient to an ICU and the length of postoperative anesthesia care unit (PACU) stay or close monitoring should be tailored according to the severity of the disease and to the risk of developing further complications.

In conclusion, patients with Takayasu arteritis vary greatly in terms of clinical presentation; thus, meticulous preoperative evaluation, intraoperative planning, and postoperative management are necessary. We have presented here a case of a young male with an incidental diagnosis of Takayasu arteritis who had undergone uneventful surgery under general anesthesia aided by a basic understanding of the uncommon disease.

\section{Acknowledgements}

The authors thank the patient for giving permission for publishing this case.

\section{Conflict of Interest}

None declared.

\section{References}

1. Seyahi E (2017) Takayasu arteritis: An update. Curr Opin Rheumatol 29: 51-56.

2. Perera AH, Mason JC, Wolfe JH (2013) Takayasu arteritis: Criteria for surgical intervention should not be ignored. International Journal of Vascular Medicine.

3. Keser G, Direskeneli H, Aksu K (2014) Management of takayasu arteritis: A systematic review. Rheumatology (Oxford) 53: 793-801.

4. Shrestha GS, Shrestha N, Shrestha BK, et al. (2015) Anaesthetic management of patients with takayasu's arteritis for open cholecystectomy. JNMA J Nepal Med Assoc 53: 144-147.

5. Ioscovich A, Gislason R, Fadeev A, et al. (2008) Peripartum anesthetic management of patients with Takayasu's arteritis: Case series and review. Int J Obstet Anesth 17: 358-364.

6. Karaca S, Akgun I (2002) Epidural anaesthesia for arthroscopic knee surgery in a patient with Takayasu's arteritis. Eur J Anaesthesiol 19: 528-529.

7. Kathirvel S, Chavan S, Arya VK, et al. (2001) Anesthetic management of patients with Takayasu's arteritis: A case series and review. Anesth Analg 93: 60-65.

8. Gupta D, Arora N (2019) Takayasu arteritis: Anaesthetic implications in caesarean section. J Clin Anesth 58: 18-19.

9. Kassa MW, Benti TM, Bedada AG (2018) Successful spinal anaesthesia for caesarean section in an African patient with Takayasu's arteritis. Pan Afr Med J 30: 281.

10. Xiao W, Wang T, Fu W, et al. (2016) Regional cerebral oxygen saturation guided cerebral protection in a parturient with Takayasu's arteritis undergoing cesarean section: A case report. J Clin Anesth 33: 168-172.

11. Gaba P, Saxena K, Dua C (2008) Takayasu's arteritis: Anaesthetic implications and role of ILMA for airway management. Indian Journal of Anaesthesia 52: 858.

12. Wang PK, Luo BH, Chen A, et al. (2005) Anesthetic considerations in Takayasu's arteritis-a case report. Tzu Chi Med J 18: 53-56.

13. Numano $F(2002)$ The story of Takayasu arteritis. Rheumatology 41: 103-106.

14. Watanabe Y, Miyata T, Tanemoto K (2015) Current clinical features of new patients with takayasu arteritis observed from cross-country research in Japan: Age and sex specificity. Circulation 132: 1701-1709.

15. Numano F, Kobayashi $Y$ (1999) Takayasu arteritis--beyond pulselessness. Intern Med 38: 226-232.

16. Cuenza LR, Grayda JM, Natividad TEL, et al. (2014) Three vessel disease with left main involvement: $A$ rare manifestation of Takayasu's Arteritis. GSTF Journal of Advances in Medical Research (JAMR) 1: 1-5.

17. Johnston SL, Lock RJ, Gompels MM (2002) Takayasu arteritis: A review. J Clin Pathol 55: 481-486.

18. Abola M (2016) Takayasu arteritis: Patient profile and outcomes.

19. Ohigashi H, Haraguchi G, Konishi M, et al. Improved prognosis of 
Citation: Ongaigui C, Anasarias RB (2020) Considerations for Takayasu Arteritis Undergoing General Anesthesia in a Resource-Limited Setting: Case Report and Literature Review. J Clin Anesth Pain Manag 4(1):88-91

Takayasu arteritis over the past decade--comprehensive analysis of 106 patients. Circ J 76: 1004-1011.

20. Arnaud L, Haroche J, Limal N, et al. (2010) Takayasu arteritis in France: A single-center retrospective study of 82 cases comparing white, North African, and black patients. Medicine 89: 1-17.

21. Hemlata, Kishore K (2014) Concomitant takayasu arteritis and cushing syndrome in a child undergoing open adrenalectomy: An anaesthetic challenge. Indian J Anaesth 58: 467-469.

22. Brunner J, Feldman BM, Tyrrell PN, et al. (2010) Takayasu arteritis in children and adolescents. Rheumatology (Oxford) 49: 1806-1814.

23. Ishikawa K (1988) Diagnostic approach and proposed criteria for the clinical diagnosis of Takayasu's arteriopathy. J Am Coll Cardiol 12: 964-972.

24. Arend WP, Michel BA, Bloch DA, et al. (1990) The American College of Rheumatology 1990 criteria for the classification of Takayasu arteritis. Arthritis Rheum 33: 1129-1134.

25. Khandelwal M, Lal N, Fischer RL, et al. (2009) Takayasu arteritis and pregnancy: Case report and review of the literature. Obstetrical \& Gynecological Survey 64: 258-272.

26. Kiran S, Bhardwaj M, Kaur K (2012) Anaesthetic management in a parturient with Takayasu arteritis. Egyptian Journal of Anaesthesia 28: 287-289.

27. Dutta B, Pandey R, Darlong V, et al. (2010) Low-dose spinal anaesthesia for a parturient with Takayasu's arteritis undergoing emergency caesarean section. Singapore Med J 51: e111-e113.

28. Khan AM, Kapoor MC, Puri A (2015) Cesarean section under spinal anesthesia in a patient of Takayasu's arteritis. Journal of Obstetric Anaesthesia and Critical Care 5: 90-92.

29. Narasimha PK, Chaudhuri S, Joseph TT (2013) Utility of intra-operative ultrasound in choosing the appropriate site for blood pressure monitoring in Takayasu's arteritis. Indian J Anaesth 57: 66-68.

30. Yoshida M, Yamamoto T, Shiiba S, et al. (2016) Anesthetic management of a patient with Takayasu Arteritis. Anesth Prog 63: 31-33.

31. Lee EH, Choi E, Ahn W (2013) Application of cerebral oximetry for a parturient with Takayasu's arteritis undergoing cesarean section-a case report. Korean J Anesthesiol 65: 158-162.

32. Clark AG, Al-Qatari M (1998) Anaesthesia for caesarean section in Takayasu's disease. Canadian Journal of Anaesthesia 45: 377.

33. Gozal Y, Ginosar Y, Gozal D (1995) Combined general and epidural anesthesia for a patient with Takayasu's Arteritis. Case report. Reg Anesth 20: 246-248.

34. Fawcett WJ, Razis PA, Berwick EP (1993) Post-operative cerebral infarction and Takayasu's disease. Eur J Anaesthesiol 10: 33-35.

35. Kamdem F, Kenmegne C, Hamadou B, et al. (2018) Multiple cerebral infarction revealing Takayasu's disease: A case report in a 32-year-old man from Cameroon, sub-Saharan Africa. Clin Case Rep 6: 569-573.

36. Bhardwaj N, Babu R, Behra A (2009) Anaesthetic management of a pregnant patient with Takayasu's disease undergoing abdominal aortic aneurysm repair. Int J Obstet Anesth 18: 392-395.

37. Tiwari AK, Tomar GS, Chadha M, et al. (2011) Takayasu's arteritis: Anesthetic significance and management of a patient for cesarean section using the epidural volume extension technique. Anesthesia, Essays and Researches 5: 98-101.

38. Leal PdC, Silveira FFM, Sadatsune EJ, et al. (2011) Takayasus's arteritis in pregnancy. Case report and literature review. Rev Bras Anestesiol 61: 482-485.

39. Jain D, Rojha M, Aggarwal M, et al. (2012) Indian origin Takayasu's arteritis: A case report with a brief review of clinical management. Anaesthesia, Pain \& Intensive Care 16: 192-194.

40. Meikle A, Milne B (1997) Extreme arterial blood pressure differentials in a patient with Takayasu's arteritis. Can J Anaesth 44: 868-871.

41. Chawla R, Kumarvel V, Girdhar K, et al. (1992) Can pulse oximetry be used to measure systolic blood pressure? Anesth Analg 74: 196-200.

42. Winn HN, Setaro JF, Mazor M, et al. (1988) Severe Takayasu's arteritis in pregnancy: The role of central hemodynamic monitoring. Am J Obstet Gynecol 159: 1135-1136.

43. Ghanem MT, Ahmed FI (2017) GlideScope versus McCoy laryngoscope: Intubation profile for cervically unstable patients in critical care setting. Egyptian Journal of Anaesthesia 33: 103-106.

44. Uchida T, Hikawa Y, Saito Y, et al. (1997) The McCoy levering laryngoscope in patients with limited neck extension. Can J Anaesth 44: 674-676.

45. Durga P, Sahu BP (2014) Neurological deterioration during intubation in cervical spine disorders. Indian J Anaesth 58: 684-692.

DOI: $10.36959 / 377 / 334$

Copyright: (c) 2019 Ongaigui C, et al. This is an open-access article distributed under the terms of the Creative Commons Attribution License, which permits unrestricted use, distribution, and reproduction in any medium, provided the original author and source are credited. 